\title{
Homotypic FADD interactions through a conserved RXDLL motif are required for death receptor-induced apoptosis
}

\author{
JR Muppidi ${ }^{1,2,3}$, AA Lobito ${ }^{1}$, M Ramaswamy ${ }^{1}$, JK Yang ${ }^{4}$, \\ $\mathrm{L} \mathrm{Wang}{ }^{4}, \mathrm{H} \mathrm{Wu}{ }^{4}$ and RM Siege $\mathrm{l}^{*, 1}$ \\ 1 Immunoregulation Unit, Autoimmunity Branch, NIAMS, NIH, Bethesda, MD \\ 20892, USA \\ 2 Department of Pharmacology, Toxicology and Therapeutics, University of \\ Kansas Medical Center, Kansas City, KS 66160, USA \\ 3 HHMI-NIH, Research Scholoars Program, Bethesda, MD 20814, USA \\ ${ }^{4}$ Department of Biochemistry, Weill Medical College of Cornell University, New \\ York, NY 10021, USA \\ * Corresponding author: RM Siegel, NIAMS, NIH, 9000 Rockville pk, Bldg 10, \\ Room 9N238, Bethesda, MD 20892, USA. Tel: +1 301496 3761; \\ Fax: + 1301480 3880; E-mail: rsiegel@ nih.gov
}

Received 05.8.05; revised 18.11.05; accepted 02.12.05; published online 20.1.06 Edited by D Vaux

\begin{abstract}
Death receptors in the TNF receptor superfamily signal for apoptosis via the ordered recruitment of FADD and caspase-8 to a death-inducing signaling complex (DISC). However, the nature of the protein-protein interactions in the signaling complex is not well defined. Here we show that FADD self-associates through a conserved RXDLL motif in the death effector domain (DED). Despite exhibiting similar binding to both Fas and caspase-8 and preserved overall secondary structure, FADD RDXLL motif mutants cannot reconstitute FasL- or TRAILinduced apoptosis and fail to recruit caspase-8 into the DISC of reconstituted FADD-deficient cells. Abolishing self-association can transform FADD into a dominant-negative mutant that interferes with Fas-induced apoptosis and formation of microscopically visible receptor oligomers. These findings suggest that lateral interactions among adapter molecules are required for death receptor apoptosis signaling and implicate self-association into oligomeric assemblies as a key function of death receptor adapter proteins in initiating apoptosis.

Cell Death and Differentiation (2006) 13, 1641-1650.

doi:10.1038/sj.cdd.4401855; published online 20 January 2006
\end{abstract}

Keywords: Fas; apoptosis; FADD; oligomerization; TNF receptor

Abbreviations: DISC, death-inducing signaling complex; DD, death domain; DED, death effector domain; SPOTS, signaling protein oligomeric transduction structures; DEF, death effector filaments

\section{Introduction}

The receptors for FasL and TRAIL directly activate apoptosis pathways critical for lymphocyte homeostasis, self-tolerance and tumor cell death. Fas and the TRAIL receptors DR4 and DR5 are TNFR superfamily members containing a conserved death domain (DD). Receptor ligation triggers recruitment of the DD in the adapter protein FADD to the receptor through known interaction surfaces in the two DDs. ${ }^{1-3}$. In addition to a $\mathrm{DD}, \mathrm{FADD}$ also contains an $\mathrm{N}$-terminal death effector domain (DED) that is structurally similar to the DD. ${ }^{4}$ The DED of FADD is known to recruit the procaspase-8 (FLICE/MACH-1) to the death-inducing signaling complex (DISC) via interactions with the tandem DEDs at the $\mathrm{N}$-terminus of caspase- $8 .^{5}$ The complex of the receptor, FADD, and caspase- 8 is referred to as the death-inducing signal complex (DISC). ${ }^{6,7}$

The DISC is thought to initiate the caspase cascade and apoptosis by inducing proximity between caspase-8 molecules. $^{8-10}$ More recent experiments have shown that in vitro, caspase dimerization is necessary for caspase activation and precedes caspase autoprocessing. ${ }^{11,12}$ However, the stoichiometry of FADD and caspase-8 in the DISC, and how receptor ligation causes caspase-8 activation is not clear. Recent data have highlighted the importance of receptor oligomerization in triggering efficient Fas-induced apoptosis. TNF-family receptors can be preassociated prior to ligand binding in submicroscopic assemblies, ${ }^{13,14}$ and we have recently identified microscopically visible microclusters of Fas termed signaling protein oligomeric transduction structures (SPOTS) that can be seen within minutes of ligand binding prior to caspase activation. ${ }^{15}$ Receptor capping and internalization follows SPOTS formation, and are also important in Fas-induced apoptosis. ${ }^{16,17}$ FADD is required for formation of SPOTS, suggesting that lateral interactions between FADD in receptor-signaling complexes may be important in transducing an efficient death signal.

The FADD protein has the potential to be highly oligomeric. FADD aggregates in vitro, ${ }^{4}$ and transfected FADD in mammalian cells forms large filamentous structures termed death effector filaments (DEF). ${ }^{18}$ Site-directed mutagenesis experiments have shown that residues in a region designated hydrophobic patch 1 , centered around residue $\mathrm{F} 25$, are important for interactions between FADD and caspase-8 (Figure 1a). ${ }^{4}$ In addition, a basic surface patch in the third $\alpha$-helix of the DED of FADD was shown to be important in binding both cellular FLICE-like inhibitory protein (c-FLIP) and caspase-8. ${ }^{19}$ A region responsible for FADD self-association has not been determined. In mutagenesis studies of the DED of the apoptosis inhibitor viral FLICE-like inhibitory protein (v-FLIP) MC159, hydrophobic patch 1 was also found to be important for interactions with FADD and caspase-8, but another domain was also found in the C-terminal portion of the DED that affected its apoptosis inhibitory function without blocking interactions with FADD or caspase-8. ${ }^{20}$ This RXDLL motif is highly conserved among proteins containing a DED, and in proteins containing tandem DED's this motif is more 
a

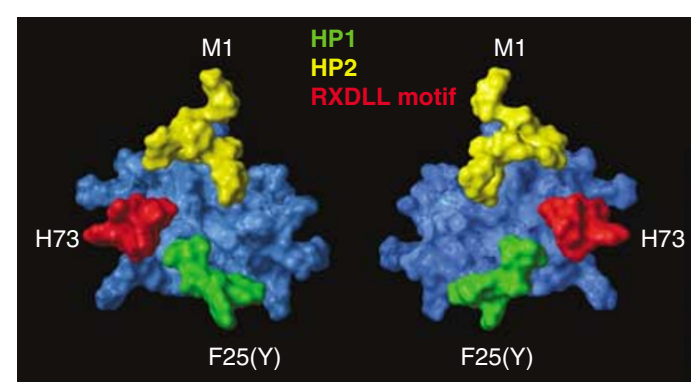

b
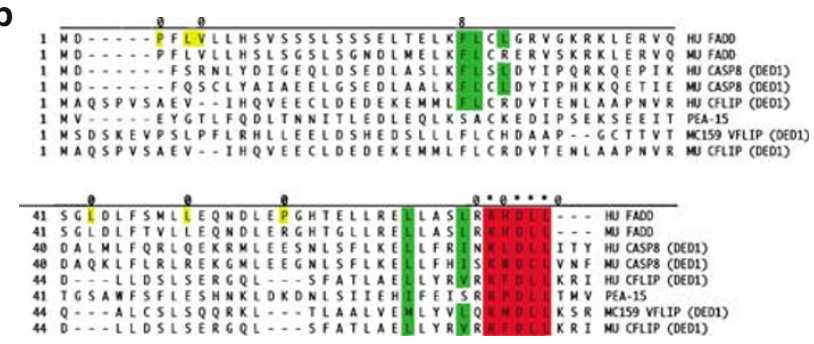

C
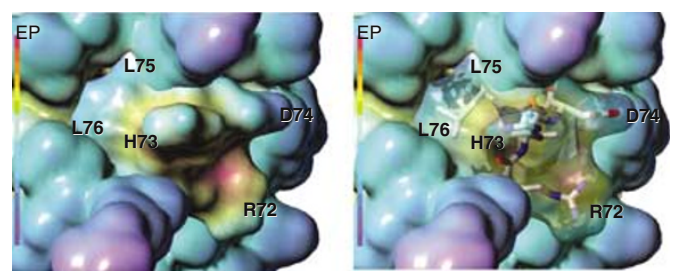

d
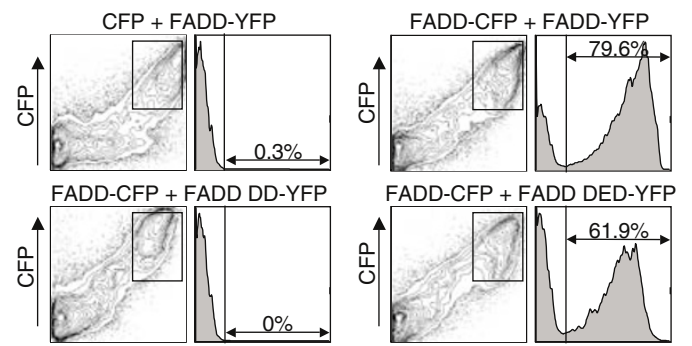

FADD DD-CFP + FADD DD-YFP
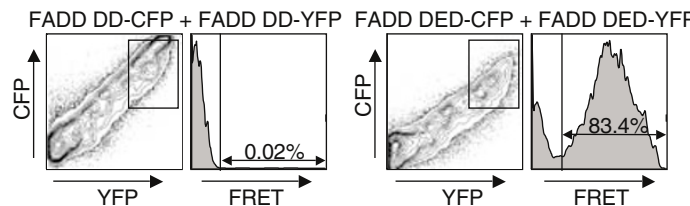

Figure 1 FADD self-association is mediated by its death effector domain (DED). (a) NMR structure of the DED of FADD. RXDLL motif (red), HP1 (green) and HP2 (yellow) are shown. (b) sequence alignment of death effector domains showing conserved residues predicted to be involved in hydrophobic patch 1 (green), hydrophobic patch 2 (yellow), and the RXDLL motif (red). The asterisks ${ }^{*}$ ) above the FADD sequence indicate FADD mutants that disrupted FADD selfassociation; 8: mutants disrupting FADD association with caspase-8; 0: mutants with no discernable phenotype. (c) Electrostatic potential (EP) surface of RXDLL motif of FADD. Red indicates a positive charge and blue, negative charge. (d) 293T cells were transfected with constructs encoding full-length wild-type FADD, FADD 1-79 (FADD DED) or FADD 80-209 (FADD DD) fused to either the cyan fluorescent protein (CFP) or the yellow fluorescent protein (YFP) or CFP alone. Cells were analyzed by FACS for CFP, YFP and fluorescence resonance energy transfer (FRET) between CFP and YFP. Cells were gated as indicated on the basis of CFP and YFP expression and the amount of FRET in gated cells is shown in the histogram to the right. The percentage of FRET-positive cells is shown in each histogram. Data are representative of at least three independent experiments

conserved in the first DED (Figure 1b). The NMR structure of the DED of FADD ${ }^{4}$ shows that the R72, H73, and D74 in this motif are exposed on the surface, with $\mathrm{L} 75$ and $\mathrm{L} 76$ directly underneath (Figure 1c).

Here we show that FADD self-association is critical for its adapter protein function in death receptor apoptosis signaling. We find that FADD self-association occurs via the DED, that the FADD-FADD interface is not equivalent to the FADDcaspase-8 interface and that FADD self-association mediated by the RXDLL motif is required for death receptor-mediated apoptotic signaling and caspase-8 recruitment to the DISC.

\section{Results}

\section{FADD self-associates through the DED}

We first investigated whether self-assocation of FADD occurs and which domain of FADD may be responsible for selfassociation. Fluorescence resonance energy transfer (FRET) allows protein-protein interactions to be measured in living cells. The use of flow cytometry to measure FRET between cyan- and yellow fluorescent proteins (CFP and YFP) has allowed rapid quantitation of protein-protein interactions in living cells in a number of settings. ${ }^{13,14,21-23}$ When constructs containing full-length FADD fused to (CFP) and (YFP), were cotransfected, there was a high level of FRET among cells coexpressing both fusion proteins when compared to cells transfected with unfused CFP and FADD-YFP. FRET was completely abrogated when the DED was removed from one or both of the constructs (Figure 1d). Additionally, full-length FADD and FADD DED, but not the FADD DD, co-immunoprecipitated with full-length FADD (data not shown). These data show that FADD self-associates in living cells and that self-association is mediated by the DED.

\section{A conserved short peptide motif in the DED mediates FADD self-association}

The NMR structure of the DED of FADD contains two surface hydrophobic patches. ${ }^{4}$ Hydrophobic patch 1 (HP1, Figure 1a, green) mediates binding to caspase- 8 and consists of the residues F25, L28, L26, L66, L70. A less conserved hydrophobic patch (HP2, Figure 1a, yellow), facing opposite HP1, consists of the residues M1, P3, L5, V6, L43, L50, P57 and F82. Single and multiple alanine mutations in HP2 amino acids did not disrupt FADD self-association or function in Fas signaling (data not shown). These data suggest that FADD self-association is not mediated by HP2 and that HP2 does not participate directly in Fas apoptosis signaling.

In addition, the DED of FADD contains the sequence RHDLL from positions 72 to 76 in the 6 th alpha helix of the DED that is homologous to an RXDLL motif conserved between many DED-containing proteins (Figure 1a-c, red). In studies of this motif in the DED containing antiapoptotic viral FLIP, MC159, it was found that the double mutants of the RHDLL to AHALL or RHDAA but not point mutations in the motif were sufficient to reduce antiapoptotic activity. ${ }^{20}$ Therefore, using site-directed mutagenesis, we altered the RXDLL motif by mutating the leucines at positions 75 and 76 to alanines (RHDAA) or, alternatively, the arginine at position 
72 and the aspartic acid at position 74 to alanines (AHALL). 293T or FADD-deficient Jurkat cells (I2.1) were cotransfected with full-length wild-type (WT), RHDAA or AHALL mutant FADD constructs fused to YFP or CFP and FRET between the two molecules was analyzed by FACS. The percentage of FRET-positive cells was substantially diminished in cells transfected with RXDLL-mutant FADD in both 293T and I2.1 FADD-deficient cells (Figure $2 a$ and $b$ ). There was no rescue of the diminished FRET when AHALL-CFP and RHDAA-YFP or AHALL-YFP and RHDAA-CFP were cotransfected (data not shown), indicating that the two mutations in the RXDLL motif cannot complement each other.

In addition to the loss of FRET shown between the RXDLL mutants, co-immunoprecipitation of these mutants with WT FADD was impaired (Figure 2c). These FADD mutants were also unable to form DEF when overexpressed in COS-7 cells (Figure 2d), showing diffuse cytoplasmic staining with occasional cytoplasmic aggregates. These data show that FADD self-association is dependent on the RXDLL motif of the DED.

The failure of these FADD mutants to oligomerize in cells could be due to a generalized unfolding of the protein. If that was the case, other DED-mediated interactions, such as binding to caspase-8, may be impaired by mutations in the RXDLL motif. We therefore assessed the ability of the RXDLL mutants to interact with caspase-8. WT FADD-YFP or RXDLL mutants were cotransfected with a catalytically inactive caspase-8 CFP in 293T cells. As expected, FRET was seen between WT FADD and caspase-8. This interaction was dependent on the DED of FADD, as the FADD DD alone failed to interact with caspase-8 (Figure 3a). Unlike the nearly complete abolition of FADD-FADD interactions, the level of FRET was maintained between caspase- 8 and the AHALL FADD mutant, and was consistently increased with the RHDAA mutant (Figure 3a). Co-immunoprecipitation experiments confirmed these results, with both WT and RXDLL mutant FADD interacting with HA-tagged caspase-8 GFP in 293T cells (Figure 3b). To confirm that the FADD DED harboring RXDLL motif mutations still folded normally into the alpha-helical DD structure, we compared the circular dichroism (CD) spectra of purified monomeric FADD DED with WT FADD, AHALL, AHDLL (R72A), and RHDAA RXDLL motif sequences. The CD spectra of all of these RXDLL motif mutants completely overlapped with that of the wild-type protein, suggesting that the global folding of the FADD DED was not perturbed by the RXDLL motif mutations. This is consistent with the retained ability of these mutants to bind to caspase-8 and suggests that the RXDLL motif is likely functioning to mediate FADD-FADD interactions, and is not required for proper folding of the FADD DED.

\section{FADD self-association is critical for death receptor-mediated apoptosis}

To test whether FADD self-association is functionally important for apoptosis signaling induced by FasL and TRAIL, we used the FADD-deficient Jurkat T-cell line, I2.1, which is a

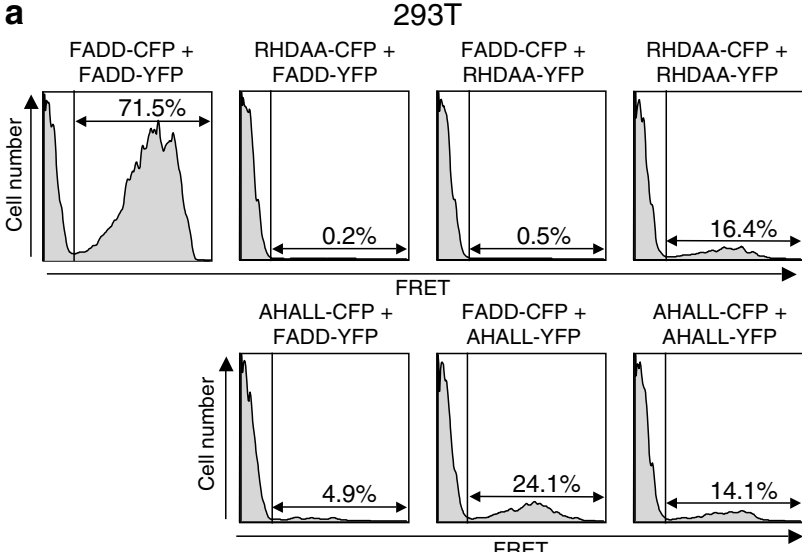

b

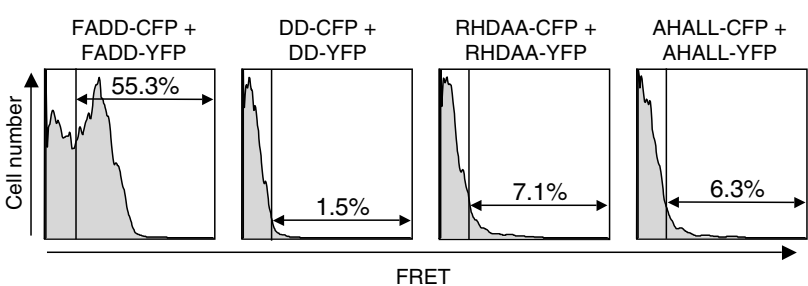

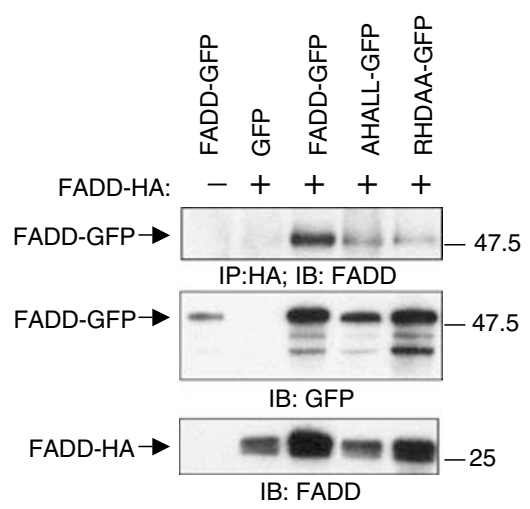

d

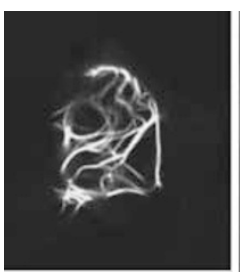

FADD-GFP

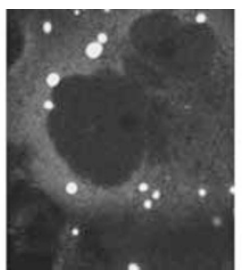

RHDAA-GFP

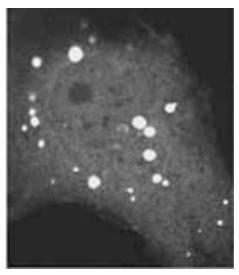

AHALL-GFP

Figure 2 FADD self-association is mediated by the RXDLL motif. (a and b) 293T cells (a) or FADD-deficient Jurkat I2.1 cells (b) were transfected with constructs encoding wild-type FADD or the RXDLL mutants of FADD (RHDAA and AHALL) fused to either CFP or YFP or CFP alone. FRET was analyzed as described above. (c) 293T cells were transfected with constructs encoding HA-FADD and WT or RXDLL mutant FADD fused to GFP. Cells were lysed and immunoprecipitated with anti-HA beads. Lysates and immunoprecipates were run on a gel and immunoblotted for both HA and GFP. (d) Cos7 cells were transfected with constructs encoding WT or RXDLL mutant FADD fused to GFP and imaged by confocal microscopy. All data are representative of at least three independent experiments 
a

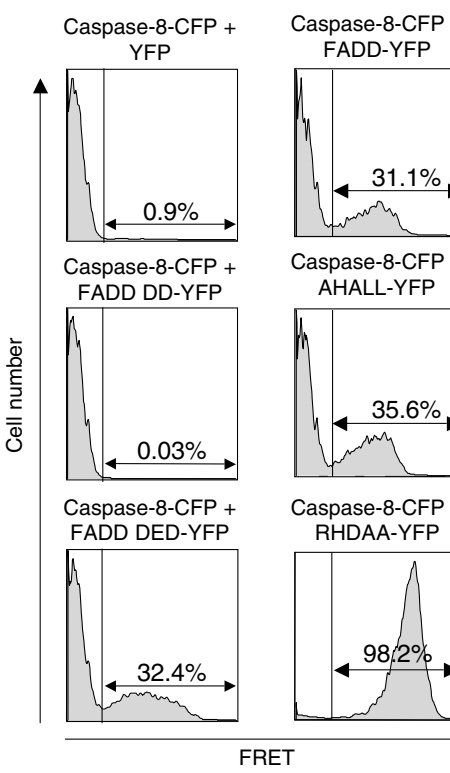

b

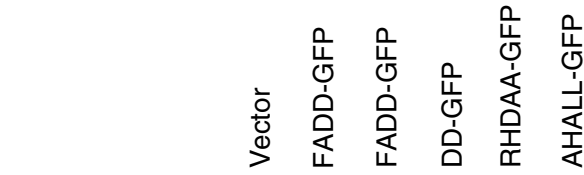

HA-Caspase-8 GFP: $+\quad+\quad+\quad+++$

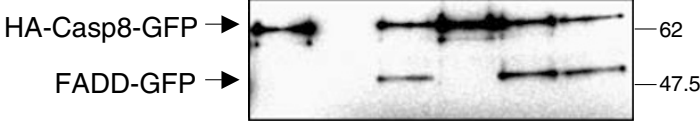

IP:HA; IB: GFP

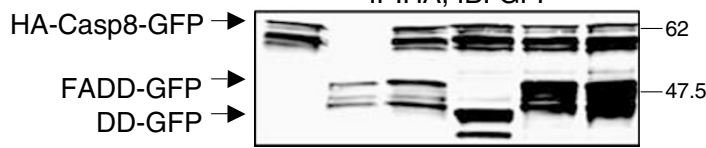

IB: GFP

C

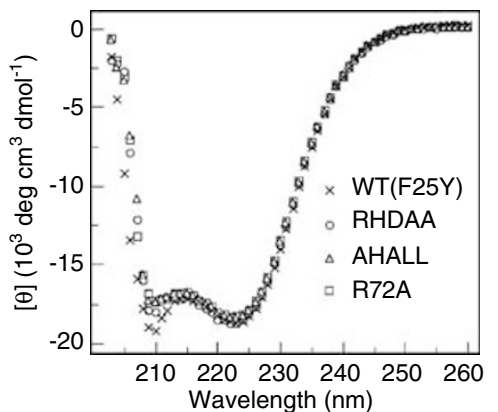

Figure 3 Caspase-8 binding and overall protein folding are preserved in FADD RXDLL motif mutants. (a) 293T cells were transfected with constructs encoding caspase-8 CFP and WT, RXDLL mutant, DD or DED FADD fused to YFP or YFP alone and FRET was analyzed as described above. (b) 293T cells were transfected with HA-caspase-8 GFP and WT, RXDLL mutant or DD FADD fused to GFP. Cells were lysed and immunoprecipitated with anti-HA beads. Lysates and immunoprecipitates were subjected to SDS-PAGE and immunoblotted for GFP. All data are representative of at least three independent experiments. (c) CD spectra of FADD F25Y and the indicated RXDLL motif mutants. The average wavelength dependence of molar ellipticity from five scans is shown, using a 5-s integration time at 1.0-nm wavelength increments

completely insensitive to apoptosis induced by these death receptor ligands. ${ }^{24}$ We transiently transfected FADDdeficient cells with WT, RXDLL mutant or DD only FADD constructs fused to YFP and stimulated these cells with FasL, anti-Fas or TRAIL to determine whether RXDLL mutants could reconstitute Fas or TRAIL receptor-mediated apoptosis in these cells. WT FADD was able to reconstitute FasL or TRAIL-mediated apoptosis in these cells, while the FADD DD was not. Significantly, the RXDLL mutants RHDAA and AHALL were almost completely deficient in their ability to reconstitute apoptosis signaling in response to FasL or TRAIL (Figure $4 a$ and b). Following FasL stimulation, cells transfected with these mutant constructs persisted in the annexin-negative gate while virtually all cells with any expression of wild-type FADD-YFP became annexin positive (Figure 4a). Although it has been reported that 12.1 expresses a smaller isoform of FADD recognized by antibodies against the DED of $F A D D,{ }^{25}$ antibodies against the FADD DD do not recognize any specific proteins in this line and it is likely that this isoform represents a truncated form of FADD lacking a functional DD. Without a DD, the truncated FADD would not be recruited to the DISC and should not influence death-receptor-induced apoptosis. In addition, the RXDLL motif mutants would likely not interact with the truncated FADD present in 12.1 cells since these mutants do not interact with WT FADD as shown in Figure 2.
Interestingly, in spite of their inability to reconstitute death receptor-mediated apoptosis signaling, transfection of RXDLL mutants resulted in levels of spontaneous death among transfected cells similar to WT FADD (upper right quadrant of untreated cells and legend, Figure 4a). Spontaneous apoptosis induced by FADD has been shown to be due to interactions with caspase- 8 mediated by residues in the FADD DED hydrophobic patch $1,4,18$ and is consistent with the ability of the RXDLL mutants to bind to caspase- $8 .{ }^{18}$ Thus, the RXDLL motif that is responsible for FADD-FADD association also appears necessary for FADD to function as death receptor adapter.

To examine the biochemical basis for the inability of RXDLL FADD mutants to function, we studied the whether FADD RXDLL mutants could support assembly of a normal Fas-FADD-Caspase-8-signaling complex. Co-immunoprecipitation experiments showed that these FADD mutants were able to interact normally with Fas in 293T cells (Figure 4c). We then reconstituted Jurkat I2.1 cells with WT FADD and RXDLL FADD mutants and studied assembly of the Fas DISC. In WT FADD transfectants, both FADD and caspase-8 were recruited to the DISC after FasL stimulation. However, in 12.1 cells transfected with either RHDAA or AHALL mutant FADD, caspase-8 was absent from the DISC despite relative preservation of FADD recruitment (Figure 4d and data not shown). These data suggest that FADD self-association is necessary for efficient 
a

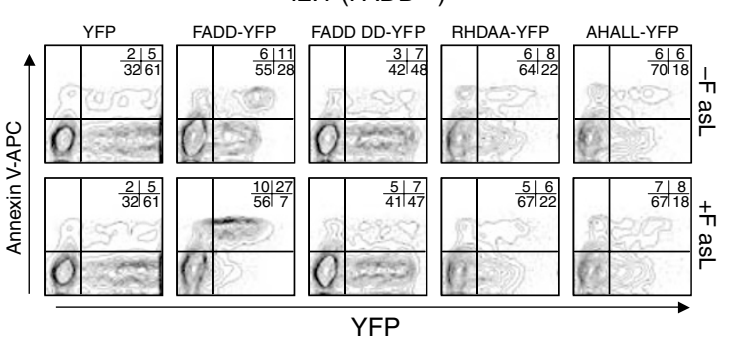

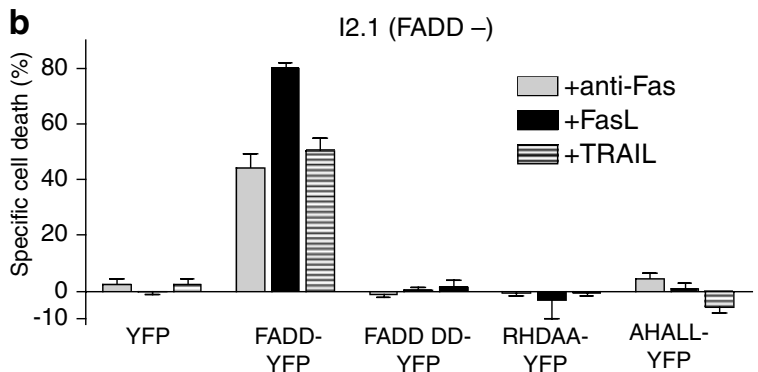

d

d $2.1($ FADD - )

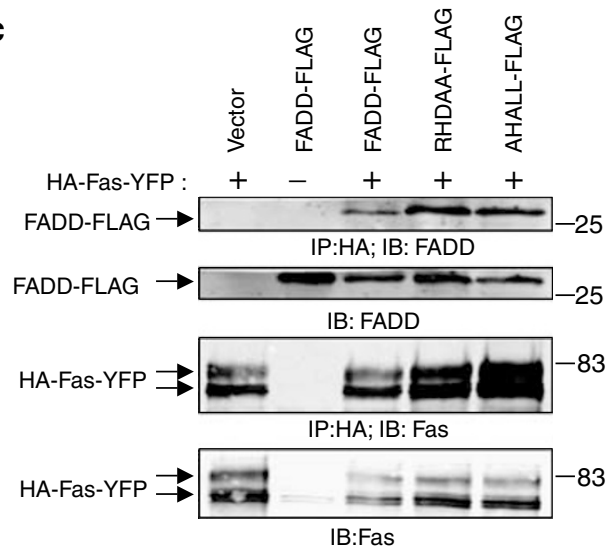

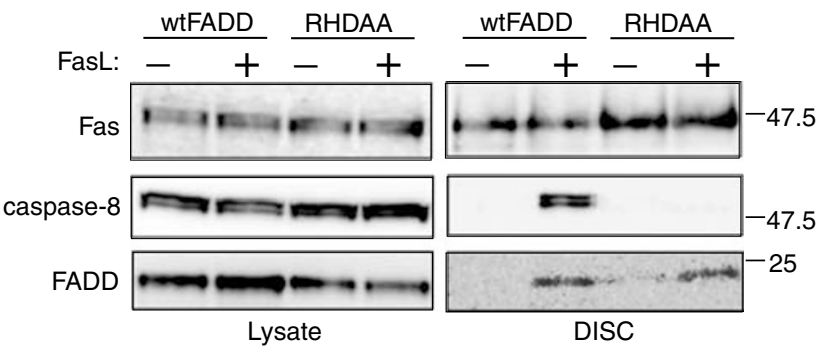

Figure 4 RXDLL mutant FADD cannot reconstitute Fas-mediated apoptosis in FADD-deficient cells. (a and $\mathbf{b})$ The FADD-deficient Jurkat cell line, I2.1, was transfected with WT, RXDLL mutant or DD FADD fused to YFP. At $16 \mathrm{~h}$ after transfection, cells were treated with anti-Fas or FasL or TRAIL for $5 \mathrm{~h}$. Cell death was analyzed by FACS with Annexin-APC and PI. Cells were gated on PI-negative cells to exclude death due to transfection and GFP-positive cells were analyzed for death by Annexin-APC. Error bars indicate S.E.M. of pooled data from 2-3 independent experiments. Prior to stimulation viability (avg. \pm S.E.M.) of transfected cells was $87.2 \pm 1.1 \%$ for YFP, $64.3 \pm 3.1 \%$ for FADD FL YFP, $80.3 \pm 1.0 \%$ for FADD DD YFP, $57.6 \pm 3.7 \%$ for RHDAA YFP and $60.9 \pm 3.7 \%$ for AHALL YFP. (c) $293 T$ cells were transfected with HA-Fas-YFP and WT or RXDLL mutant FADD. Cells were lysed and immunoprecipitated with anti-HA beads. Lysates and immunoprecipitates were run on a gel and immunoblotted for Fas and FADD. (d) I2.1 cells were transfected with WT or RXDLL mutant FADD. At $16 \mathrm{~h}$ after transfection, cells were stimulated with antiFas for $15 \mathrm{~min}$. DISC formation was analyzed by measuring recruitment of transfected FADD and endogenous caspase-8 to endogenous Fas. All data are representative of at least three independent experiments

recruitment and activation of caspase-8 to the DISC during Fas signaling.

To examine whether mutations in the RXDLL motif could transform FADD into a dominant-negative inhibitor, we transfected WT FADD and the RXDLL mutants into a number of different Fas-sensitive cell lines expressing endogenous FADD. Fas-expressing cells have been divided into two categories. In type I cell lines, the DISC is easily detectable and Fas-induced cell death cannot be blocked by overexpression of antiapoptotic Bcl-2 family molecules. In type II cell lines, the DISC is less easily detected biochemically and Fas-induced cell death can be blocked by the overexpression of antiapoptotic Bcl-2 molecules. ${ }^{26}$ In type I cell lines, Fas is preferentially localized to lipid raft microdomains, and surface receptor clustering is more rapid and prominent than in type II cell lines. ${ }^{15,27}$ We transfected Jurkat, a type II cell line, and SKW6.4, a type I cell line, with WT, RXDLL mutant or the DD of FADD fused to YFP and assessed the ability of transfected cells to undergo apoptosis in response to Fas stimulation. FADD DD-YFP inhibited Fas-induced apoptosis in Jurkat (type II) and more prominently in SKW6.4 (type I) cells. FADD constructs with mutations in the RXDLL motif inhibited Fasinduced apoptosis in SKW6.4 but not in Jurkat cells (Figure 5a and b). Since assembly and activation of the DISC has been associated with formation of visible receptor oligomers termed SPOTS ${ }^{15}$ we examined the subcellular localization of Fas in SKW 6.4 cells transfected with RXDLL mutant FADD and found that these mutants interfered with the ability of Fas to form SPOTS and receptor caps after receptor crosslinking (Figure 5c). These data show that non-self-associating FADD mutants can dominantly interfere with Fas-induced cell death in type I cells, likely by interfering with SPOTS formation mediated by endogenous FADD.

To define the limits of the RXDLL motif we created individual alanine mutants of R72, H73 and D74 in the RXDLL motif as well as the adjacent $R 71$ and $R 77$ residues. Like the more complex RHDAA and AHALL mutants, the R72A and D74A mutants showed diminished binding to wild-type FADD and did not fully reconstitute Fas-mediated apoptosis (Figure 6a and b). It should also be noted that R72A, which had greater defect in its association with WT FADD than D74A, similarly had a greater defect in reconstitution of Fas-mediated apoptosis. In contrast, R71A, H73A, and R77A mutants functioned equivalently to wild-type FADD in these assays (Figure $6 \mathrm{a}$ and b). Although a surface-exposed residue, $\mathrm{H} 73$ is not conserved in the RXDLL motif and is apparently not required for FADD function. Additionally, the R72A FADD mutant but not R71A dominantly interfered with Fas-induced 

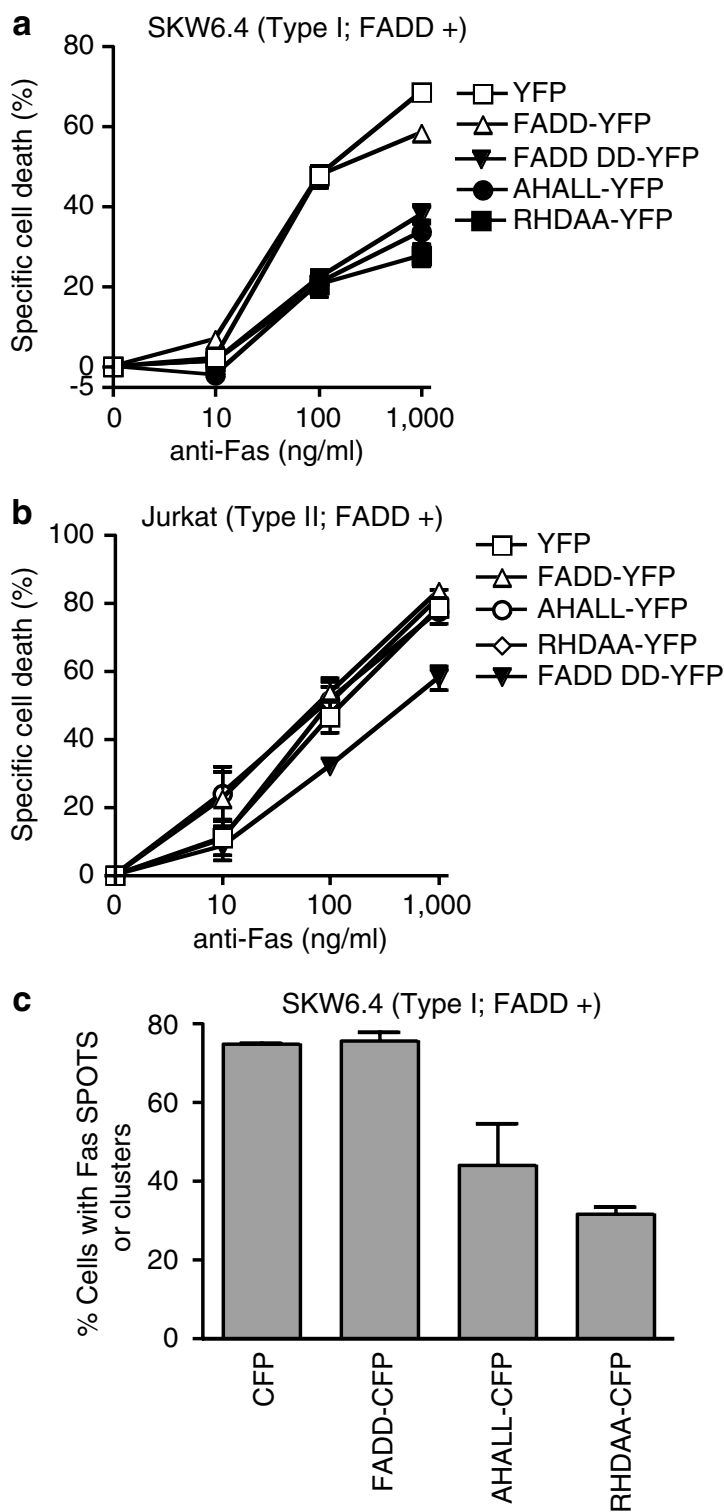

Figure 5 RXDLL mutant FADD dominantly interferes with Fas signaling and receptor clustering in type I cells. (a and b) SKW 6.4 (a) or Jurkat (b) cells were transfected with WT, RXDLL mutant or DD FADD fused to YFP. At $16 \mathrm{~h}$ after transfection, cells were treated with anti-Fas for $5 \mathrm{~h}$. Cell death was analyzed as above. Error bars indicate S.E.M. of pooled data from three independent experiments. In Jurkat cells, prior to stimulation viability (avg. \pm S.D.) of transfected cells was $90.2 \pm 1.8 \%$ for YFP, $62.7 \pm 7.8 \%$ for FADD FL YFP, $75.0 \pm 5.8 \%$ for FADD DD YFP, $56.3 \pm 10.8 \%$ for RHDAA YFP and $55.1 \pm 10.6 \%$ for AHALL YFP. In SKW 6.4 cells, prior to stimulation viability (avg. \pm S.D.) of transfected cells was $80.0 \pm 1.6 \%$ for YFP, $59.1 \pm 3.4 \%$ for FADD FL YFP, $74.5 \pm 3.1 \%$ for FADD DD YFP, $72.4 \pm 3.7 \%$ for RHDAA YFP and $65.1 \pm 1.9 \%$ for AHALL YFP. (c) SKW 6.4 cells were transfected with the indicated FADD-CFP constructs, stimulated with anti-Fas for $1 \mathrm{~h}$, fixed and analyzed for Fas localization by immmunofluorescence as described in the Materials and Methods. Cells were visually scored for SPOTS and receptor caps and the average + S.E.M percent cells with SPOTS or receptor caps are shown. All data are representative of at least three independent experiments

apoptosis when transfected into SKW 6.4 cells (Figure 6c). These data are in contrast to a report in which FADD R71A was found to be deficient in function and binding to the Fas DD. ${ }^{28}$ These results define R72, D74, L75 and L76 as key

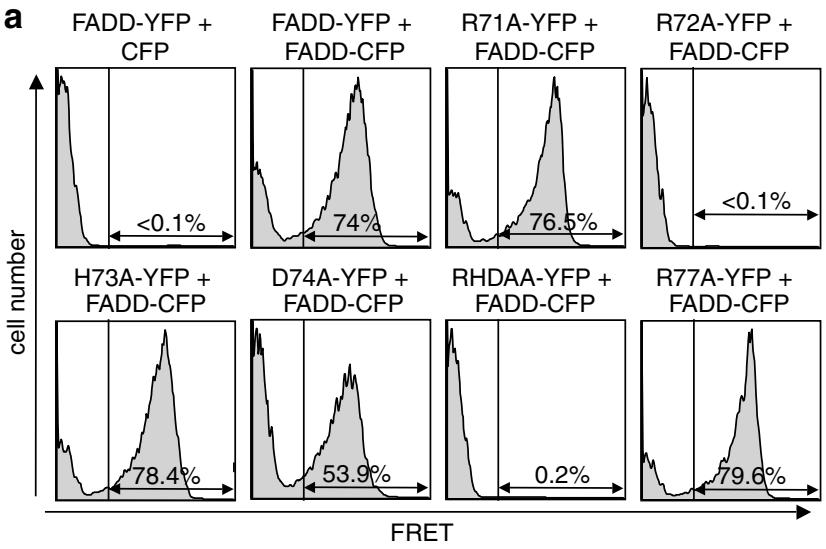

b

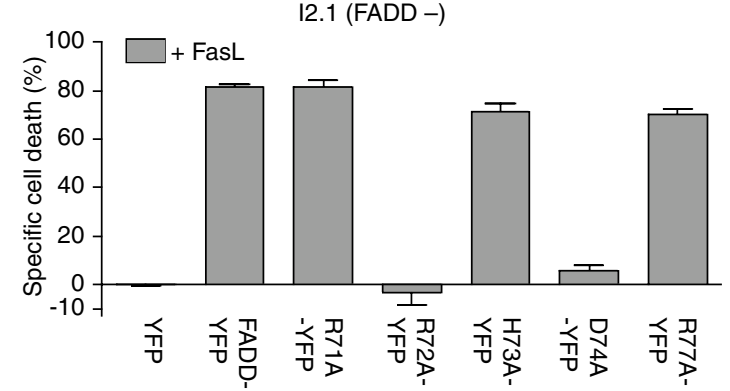

C

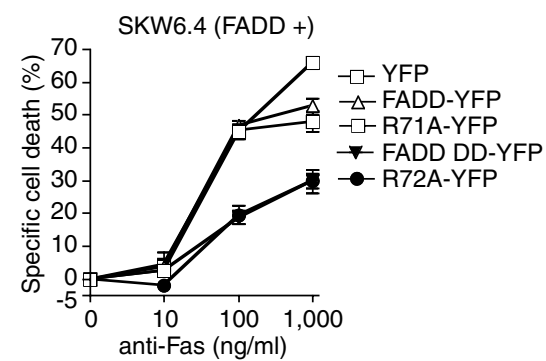

Figure 6 Deficiencies in FADD self-association and Fas signaling are specific for the RXDLL motif. (a) 293T cells were transfected with constructs encoding CFP alone or FADD-CFP and WT, R71A, R72A, H73A, D74A, RHDAA or R77A FADD fused to YFP and FRET was analyzed as described above. (b) FADDdeficient I2.1 cells were transfected with WT, R71A, R72A H73A, D74A or R77A FADD fused to YFP or YFP alone. At $16 \mathrm{~h}$ after transfection, cells were treated with FasL for $5 \mathrm{~h}$. Cell death was analyzed as in Figure $4 \mathrm{~b}$ and are averages of triplicate data from 1 to 3 experiments \pm S.E.M. Prior to stimulation viability (avg. \pm S.D.) of transfected cells was $86.4 \pm 1.1 \%$ for YFP, $50.9 \pm 3.8 \%$ for FADD FL YFP, $54.6+4.8 \%$ for R71A YFP, $48.7+6.2 \%$ for R72A YFP, $39.5 \pm 1.0 \%$ for H73A YFP, $42.7 \pm 0.7 \%$ for D74A YFP and $40.4 \pm 1.4 \%$ for R77A YFP. (c) SKW 6.4 cells were transfected with WT, R71A, R72A or DD FADD fused to YFP or YFP alone. At $16 \mathrm{~h}$ after transfection, cells were treated with anti-Fas for $5 \mathrm{~h}$. Cell death was analyzed as above. Error bars indicate S.E.M. of pooled data from 2 to 3 independent experiments. Prior to stimulation viability (avg. \pm S.D.) of transfected cells was $88.6 \pm 1.1 \%$ for YFP, $72.6 \pm 2.3 \%$ for FADD FL YFP, $84.5 \pm 1.5 \%$ for FADD DD YFP, $63.4 \pm 1.4 \%$ for R71A YFP and $68.2 \pm 0.6 \%$ for R72A YFP. All data are representative of at least two independent experiments

functional residues of the FADD-FADD-binding motif and indicate that nonconserved residues inside and outside the motif are not required.

Densitometric analysis of FADD expression in transiently transfected Jurkat cells showed that transfected FADD 
a

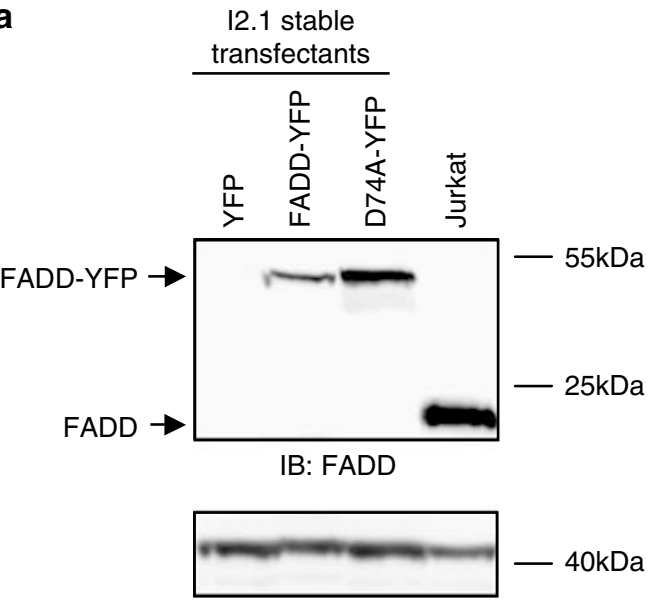

IB: Actin

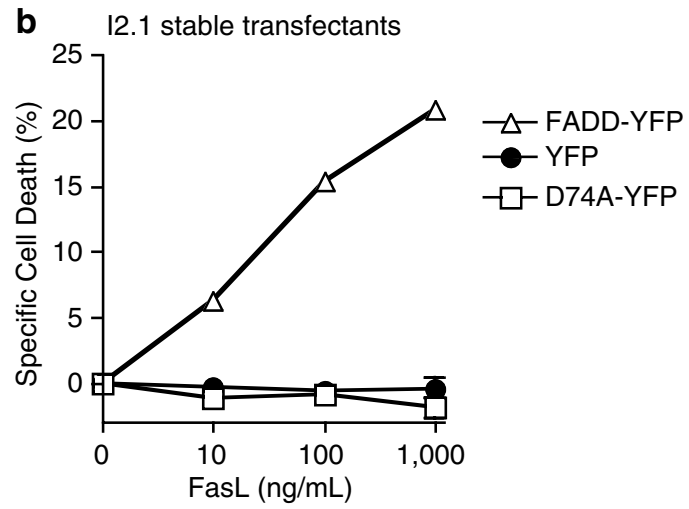

Figure 7 Stable expression of RXDLL mutant FADD in 12.1 cells fails to reconstitute Fas-mediated apoptosis. FADD-deficient 12.1 cells were stably transfected with WT or D74A FADD fused to YFP or YFP alone, as described in the Materials and Methods. (a) Immunoblots of the indicated transfectants were probed with anti-FADD and actin (b) The indicated FADD stable transfectants were treated overnight with FasL and cell viability was measured by Annexin and PI. Specific cell death was analyzed as above. Initial viability of the cells was $88 \pm 1 \%$ (YFP); $91 \pm 1 \%$ (WT FADD); and $87 \pm 2 \%$ (FADD D74A). Data are the average of two independent experiments performed in triplicate

was expressed between three and four-fold higher than endogenous FADD (data not shown). To determine whether the RXDLL motif is important for FADD expressed at more physiological levels we generated stable cell lines of FADDdeficient I 2.1 Jurkat cells expressing YFP alone, FADD-YFP or D74A FADD-YFP. The amount of FADD expressed by these cells was found to be less than or similar to that of endogenous FADD expressed by Jurkat cells (Figure 7a). At these levels, WT FADD still reconstituted sensitivity to FasLinduced apoptosis while a line expressing slightly higher levels of D74A FADD mutant did not (Figure 7c). These data indicate that critical residues in the RXDLL motif are required for FADD function as an apoptosis adapter at physiological levels of FADD.

\section{Discussion}

Our results show that self-association mediated by the RXDLL motif within the DED of FADD is critical for death receptor signaling. We have found that the RXDLL motif is required for FADD self-association, but not association with Fas or caspase-8. An intact RXDLL motif is required to reconstitute death receptor-mediated apoptosis and the DISC in FADDdeficient cells. Finally, mutation of the RXDLL motif can convert FADD into a dominant-negative inhibitor of Fasinduced apoptosis.

FADD mutations in the RXDLL motif appear to specifically inhibit FADD-FADD homotypic interactions rather than 'upstream' interactions with Fas or 'downstream' interactions with caspase-8. Because binding to Fas and Casapase- 8 and the CD spectra of the purified DED are preserved, it is unlikely that these mutations impair global folding of the protein. Rather, a specific protein-protein interaction surface is likely disrupted. Our results implicate R72 and D74 as key surface residues that mediate FADD self-association, with $\mathrm{L} 75$ and L76 forming the hydrophobic 'floor' of the motif. In accord with its nonconserved status, mutation of the $\mathrm{H} 73$ residue did not affect FADD function. Interestingly, analysis of the NMR structure of FADD shows that R72 and D74 contribute to alternating surface acidic and basic patches (Figure 1). It is likely that the RXDLL motif binds to its counterpart on another DED, because if another molecular surface was required, then RXDLL motif mutants should be able to interact with the RXDLL motif in WT FADD through that surface. However, this was not the case, as shown in Figure 2, RXDLL mutants were as severely impaired in binding WT FADD as themselves.

FADD self-association appears to function by promoting the formation of receptor oligomers on the cell surface after receptor ligation. We previously reported that these structures, referred to as SPOTS, are critical for full caspase activation in the DISC. ${ }^{15}$ We propose that the RXDLL motif allows FADD to self-associate following its recruitment to the DISC. RXDLL-mediated FADD self-association may then drive the recruitment of caspase- 8 to the DISC by increasing the avidity of the complex for caspase- 8 . The RXDLL motif is necessary but likely not sufficient for self-association since the motif is conserved in DED of the antiapoptotic MC159 vFLIP protein, which does not self-associate or form death-effector filaments, and acts as a dominant-negative inhibitor of FasLand TRAIL-induced apoptosis. ${ }^{15,20}$

RXDLL mutant FADD and FADD DD are more potent dominant-negative inhibitors of Fas signaling in type I cell lines such as SKW 6.4 than in type II cell lines such as Jurkat. These differences may arise because receptor oligomerization is more important for proximal signaling in type I cell lines. We have previously found that in type I but not type II cell lines, a proportion of Fas localizes to detergent-resistant lipid rafts. Lipid raft localization reduces the requirement for crosslinking of agonistic anti-Fas antibodies that induced apoptosis. ${ }^{27}$ Disruption of lipid rafts in type I cells with cyclodextrins inhibits ligand-induced SPOTS formation and receptor capping (JRM and RMS, unpublished observations). Thus in type II cells, the increased Fas crosslinking required to produce an apoptotic response may overcome the dominant-negative effect of mutations that inhibit FADD self-association.

The transformation of FADD from an obligate adapter protein to dominant-negative inhibitor reveals an important principle in death receptor signaling: that in addition to binding up and downstream signaling molecules, adapter proteins 
may need to self-associate to allow correct assembly and activation of caspase-8 in the DISC. Whereas, both RXDLL mutants that lack the ability to self-associate and FADD mutants lacking the ability to bind caspase-8, such as F25G, fail to reconstitute Fas-induced apoptosis, only RXDLL mutant FADD can dominantly interfere with Fas-induced apoptosis (JRM and RMS, unpublished observations). These data suggest that blocking the ability of FADD to self-associate and form an 'array' of signaling complexes may be an efficient mechanism for blocking death-receptor-induced apoptosis. Indeed, based on structural studies it has recently been proposed that the antiapoptotic activity of the DED-containing viral FLIP molecule MC159 is dependent on its ability to inhibit FADD self-association. ${ }^{29}$

Receptors in the TNF receptor superfamily and the toll-like receptor/IL-1 receptor superfamily signal through the recruitment of modular adapter proteins that associate with intracellular effector molecules to trigger downstream events. Rather than being a linear sequence of 'vertical' recruitment events, our results indicate that self-association of adapter proteins can mediate 'horizontal' interactions that amplify the initial ligand-receptor interaction signal and trigger more efficient recruitment of cytoplasmic effectors. Signal amplification through horizontal adapter protein interactions may produce increased sensitivity to changes in ligand concentration that can fine tune cellular responses. ${ }^{30}$ This may be particularly important for receptors that mediate irreversible outcomes such as programmed cell death. The membranebound signaling complexes promoted by FADD oligomerization appear to be critical for recruitment and processing of caspase-8. These complexes may be similar to cytoplasmic high-molecular weight complexes that have been found to be critical for activation of other caspases such as the apoptosome for caspase-9, the PIDDosome for caspase-2 and the inflammasome for caspase-1. ${ }^{31-33}$ RXDLL-mediated FADD self-association may also be an attractive target for therapeutic intervention in apoptosis signaling pathways that depend on FADD and other DED-containing proteins.

\section{Materials and Methods \\ Cells}

FADD-deficient Jurkat 12.1, Jurkat A3, SKW 6.4, Cos7 and 293T cells were obtained from ATCC. Jurkat and SKW 6.4 cells were cultured in RPMI supplemented with fetal calf serum $(10 \%)$, penicillin $(5 \mu \mathrm{g} / \mathrm{ml})$ and streptomycin $(5 \mu \mathrm{g} / \mathrm{ml})$. Cos7 and 293T cells were cultured in DMEM supplemented with fetal calf serum $(10 \%)$, penicillin $(5 \mu \mathrm{g} / \mathrm{ml})$ and streptomycin $(5 \mu \mathrm{g} / \mathrm{ml})$. All cells were grown in $5 \% \mathrm{CO}_{2}$ at $37^{\circ} \mathrm{C}$.

\section{Antibodies and reagents}

The Fas C20 antibody was obtained from Santa Cruz Antibodies (Santa Cruz, CA, USA). The FADD antibody was obtained from BD Transduction labs and the caspase-8 $\mathrm{C} 15$ antibody was obtained from Dr. M Peter (University of Chicago, Chicago, IL, USA). Anti-GFP was obtained from Roche. Anti-HA antibody and anti-HA affinity matrix was obtained from Covance.

\section{Plasmids and mutagenesis}

FADD was cloned into pEGFP-N1, pECFP-N1 and pEYFP-N1 using Pfu Turbo (Stratagene) polymerase chain reaction using primers flanking either end of FADD. FADD was mutagenized by Quick Change sitedirected mutagenesis (Stratagene) using primers specific for the desired mutations. Mutations were confirmed by sequencing.

\section{FRET analysis}

In all, $1 \times 10^{6} 293$ T cells per well were seeded in six-well dishes and transfected with expression vectors encoding a CFP fusion protein $(500 \mathrm{ng})$ and a YFP fusion protein $(500 \mathrm{ng})$ using Fugene 6 transfection reagent (Roche). Cells were harvested $24 \mathrm{~h}$ after transfection, and analyzed on a CyAn flow cytometer (Cytomation). Excitation lasers and filters for detection channels were: a $405 \mathrm{~nm}$ UV diode laser and a 450 / $50 \mathrm{~nm}$ bandpass filter for CFP; an $488 \mathrm{~nm}$ diode laser and a $546 / 10 \mathrm{~nm}$ bandpass filter for YFP; and the $405 \mathrm{~nm}$ UV diode laser and a $546 / 10 \mathrm{~nm}$ bandpass filter for FRET. All three signals were detected from the same individual cells by sequentially illuminating them with the 488 and $405 \mathrm{~nm}$ lasers.

\section{CD spectroscopy}

The F25Y soluble variant of the FADD AA 1-84 was cloned into the Pet28A vector (Novagen) and site-directed mutagenesis was performed with the quickchange method and confirmed by sequencing in all cases. Proteins were expressed in Escherichia coli strain BL21-CodonPlus(DE3)RIPL (Stratagene), and purified through Ni-affinity chromatography. Purified samples were desalted using HiPrep 26/10 Desalting column (Amersham Biosciences), and adjusted to a concentration of $0.69 \mathrm{mg} / \mathrm{ml}$. CD spectra were acquired at $4^{\circ} \mathrm{C}$ on an Aviv 62DS (Aviv Associates) CD spectropolarimeter with a $1 \mathrm{~mm}$ path-length cuvette. The wavelength dependence of molar ellipticity was monitored as the average of five scans, using a 5-s integration time at $1.0-\mathrm{nm}$ wavelength increments.

\section{Apoptosis induction assays}

I2.1 (FADD deficient), A3 Jurkat or SKW 6.4 cell lines were transiently transfected by electroporation at $260 \mathrm{~V}, 725 \Omega$ and $1025 \mu \mathrm{F}$ as previously described $^{2}$ using a BTX ECM 610 electroporator with constructs as indicated. At $16 \mathrm{~h}$ after transfection, cells were incubated with TRAIL $(50 \mathrm{ng} / \mathrm{ml}$; Alexis) or FasL $(100 \mathrm{ng} / \mathrm{ml}$; Alexis) plus anti-FLAG or antihuman Fas APO1-3 ( $1 \mu \mathrm{g} / \mathrm{ml}$; Kamiya Biomedical, Seattle, WA, USA) plus anti-lgG3 for $4-6 \mathrm{~h}$. Cells were stained with Annexin V-APC (BD Pharmingen) and propidium iodide (PI) according to manufacturer's instructions. Data were collected on a FACS Calibur flow cytometer (Becton Dickinson) and analyzed using FlowJo (Treestar Inc.). Cells were gated on PI-negative cells to exclude death due to electroporation, within the PI-negative gate viability of GFP-positive cells was assessed on the basis of Annexin V. Specific cell death of transfected cells was determined using the formula $(1-(\%$ viable treated $/ \%$ viable untreated $)) \times 100$. The S.E. in the mean is shown for cell death assays run in triplicate.

\section{Generation of stably transfected cell lines}

Constructs encoding YFP alone or wild-type or mutant FADD fused to YFP were linearized with Asel and transfected into the FADD-deficient Jurkat cell line, I 2.1, by electroporation as described above. Cells were then cultured in media containing G418 (1 mg/ml; Stratagene, CA, USA) for 
2 weeks. Cells were then sorted based on YFP expression using a MoFlo cell sorter (Dako Cytomation, CA, USA) and sorted YFP-positive cells were cultured in media containing G418 (1 mg/ml) for another 2 weeks to generate the stable cell lines.

\section{Immunoprecipitation and western analysis}

In all, $1 \times 10^{6} 293$ T cells were seeded in six-well plates and transfected with Fugene 6 with the indicated constructs. At $24 \mathrm{~h}$ after transfection, cells were harvested and lysed on ice in $1 \mathrm{ml}$ lysis buffer (1\% NP-40 (Sigma), $10 \%$ glycerol, Complete Protease Inhibitor (Roche), $5 \mathrm{mM}$ iodoacetic acid, $10 \mu \mathrm{M}$ zVAD (Envyme Systems Products), $150 \mathrm{mM} \mathrm{NaCl}, 15 \mathrm{mM}$ EDTA, $10 \mathrm{mM}$ Tris- $\mathrm{HCl}$ ). Alternatively, 12.1 cells were transfected by electroporation with the indicated constructs. A total of 15 transfections for each condition were pooled. At $16 \mathrm{~h}$ after transfection, cells were stimulated for $15 \mathrm{~min}$ with recombinant CD8-FasL ( $2.5 \mu \mathrm{g} / \mathrm{ml}$; Ancell) and lysed on ice in $1 \mathrm{ml}$ lysis buffer.

Lysates were immunoprecipitated with the indicated antibodies and Protein A agarose beads (Roche) or anti-HA affinity matrix beads (Covance) overnight at $4^{\circ} \mathrm{C}$. Immunoprecipitates were washed six times in lysis buffer. Lysates and immunoprecipitates were mixed with lithium dodecyl sulfate-containing loading buffer (Invitrogen) and DTT (Sigma) and incubated at $80^{\circ} \mathrm{C}$ for $20 \mathrm{~min}$. Lysates and immunoprecipitates were run on $4-12 \%$ Bis-Tris gel (Bio-Rad) and transferred to nitrocellulose and immunoblotted as indicated.

\section{Confocal microscopy}

Cos7 cells were grown on coverslip chambers (Lab-Tek) and transfected as indicated using Fugene 6 transfection reagent. At $16 \mathrm{~h}$ after transfection, cells were imaged live using an Olympus confocal microscope. Images were analyzed using Olympus and Imaris software.

\section{Quantitation of SPOTS formation}

SKW 6.4 cells were transfected by electroporation with CFP alone or WT or mutant FADD-CFP. At $16 \mathrm{~h}$ after transfection, dead cells were removed using Ficoll density gradient medium (Amersham). Cells were stimulated with $1 \mu \mathrm{g} / \mathrm{ml}$ APO1-3 plus anti-IgG3 or FasL plus anti-FLAG for $45 \mathrm{~min}$ and adhered to poly-L lysine-coated coverslips for 15-30 min. Cells were fixed on dry ice with ice-cold $100 \%$ methanol for $7 \mathrm{~min}$, followed by washing and staining of unstimulated cells with APO1-3 and staining of all cells with anti-mouse IgG-Alexa-488 (Molecular Probes) in PBS/0.01\% Tween-20/ $0.1 \%$ BSA (IFA buffer). Quantitation of surface receptor clustering in CFP. positive transfected cells was performed as previously described ${ }^{15}$ by a blinded observer and at least 50 cells were counted for every condition in an experiment. Cells with Fas SPOTS involving $>25 \%$ of the plasma membrane or in membrane caps were counted as positive.

\section{Acknowledgements}

We thank B Ahvazi for protein modeling; NIAMS and NIAID core flow cytometry facilities for technical assistance; M Lenardo, J Cohen, L Zheng and D Kastner for critically reviewing the manuscript. JRM was a Howard Hughes Medical Institute-National Institutes of Health Research Scholar and is supported by Grant number 5-T32-ES007079 from the National Institute of Environmental Health Sciences (NIEHS), NIH.

\section{References}

1. Chinnaiyan AM, O'Rourke K, Tewari M and Dixit VM (1995) FADD, a novel death domain-containing protein, interacts with the death domain of Fas and initiates apoptosis. Cell 81: 505-512

2. Martin DA, Zheng L, Siegel RM, Huang B, Fisher GH, Wang J, Jackson CE, Puck JM, Dale J, Straus SE, Peter ME, Krammer PH, Fesik S and Lenardo MJ (1999) Defective CD95/APO-1/Fas signal complex formation in the human autoimmune lymphoproliferative syndrome, type la. Proc. Natl. Acad. Sci. USA 96: 4552-4557

3. Huang B, Eberstadt M, Olejniczak ET, Meadows RP and Fesik SW (1996) NMR structure and mutagenesis of the Fas (APO-1/CD95) death domain. Nature 384: 638-641

4. Eberstadt M, Huang B, Chen Z, Meadows RP, Ng SC, Zheng L, Lenardo MJ and Fesik SW (1998) NMR structure and mutagenesis of the FADD (Mort1) death-effector domain. Nature 392: 941-945

5. Muzio M, Chinnaiyan AM, Kischkel FC, O'Rourke K, Shevchenko A, Ni J, Scaffidi C, Bretz JD, Zhang M, Gentz R, Mann M, Krammer PH, Peter ME and Dixit VM (1996) FLICE, a novel FADD-homologous ICE/CED-3-like protease, is recruited to the CD95 (Fas/APO-1) death-inducing signaling complex. Cell 85: 817-827

6. Kischkel FC, Hellbardt S, Behrmann I, Germer M, Pawlita M, Krammer PH and Peter ME (1995) Cytotoxicity-dependent APO-1 (Fas/CD95)-associated proteins form a death-inducing signaling complex (DISC) with the receptor. EMBO J. 14: 5579-5588

7. Peter ME and Krammer PH (2003) The CD95(APO-1/Fas) DISC and beyond. Cell Death Differ. 10: 26-35

8. Martin DA, Siegel RM, Zheng L and Lenardo MJ (1998) Membrane oligomerization and cleavage activates the caspase-8 (FLICE/MACHalpha1) death signal. J. Biol. Chem. 273: 4345-4349

9. Yang X, Chang HY and Baltimore D (1998) Autoproteolytic activation of procaspases by oligomerization. Mol. Cell 1: 319-325

10. Muzio M, Stockwell BR, Stennicke HR, Salvesen GS and Dixit VM (1998) An induced proximity model for caspase-8 activation. J. Biol. Chem. 273: 2926-2930

11. Donepudi M, Sweeney AM, Briand $C$ and Grutter MG (2003) Insights into the regulatory mechanism for caspase-8 activation. Mol. Cell 11: 543-549

12. Boatright KM, Renatus M, Scott FL, Sperandio S, Shin H, Pedersen IM, Ricci JE, Edris WA, Sutherlin DP, Green DR and Salvesen GS (2003) A unified model for apical caspase activation. Mol. Cell 11: 529-541

13. Siegel RM, Frederiksen JK, Zacharias DA, Chan FK, Johnson M, Lynch D, Tsien RY and Lenardo MJ (2000) Fas preassociation required for apoptosis signaling and dominant inhibition by pathogenic mutations. Science 288 : 2354-2357

14. Chan FK, Chun HJ, Zheng L, Siegel RM, Bui KL and Lenardo MJ (2000) A domain in TNF receptors that mediates ligand-independent receptor assembly and signaling. Science 288: $2351-2354$

15. Siegel RM, Muppidi JR, Sarker M, Lobito A, Jen M, Martin D, Straus SE and Lenardo MJ (2004) SPOTS: signaling protein oligomeric transduction structures are early mediators of death receptor-induced apoptosis at the plasma membrane. J. Cell Biol. 167: 735-744

16. Cremesti A, Paris F, Grassme H, Holler N, Tschopp J, Fuks Z, Gulbins E and Kolesnick R (2001) Ceramide enables fas to cap and kill. J. Biol. Chem. 276: 23954-23961

17. Algeciras-Schimnich A, Shen L, Barnhart BC, Murmann AE, Burkhardt JK and Peter ME (2002) Molecular ordering of the initial signaling events of CD95. Mol. Cell. Biol. 22: 207-220

18. Siegel RM, Martin DA, Zheng L, Ng SY, Bertin J, Cohen J and Lenardo MJ (1998) Death-effector filaments: novel cytoplasmic structures that recruit caspases and trigger apoptosis. J. Cell Biol. 141: 1243-1253

19. Kaufmann M, Bozic D, Briand C, Bodmer JL, Zerbe O, Kohl A, Tschopp J and Grutter MG (2002) Identification of a basic surface area of the FADD death effector domain critical for apoptotic signaling. FEBS Lett. 527: 250-254

20. Garvey TL, Bertin J, Siegel RM, Wang GH, Lenardo MJ and Cohen J (2002) Binding of FADD and caspase-8 to molluscum contagiosum virus MC159 v-FLIP is not sufficient for its antiapoptotic function. J. Virol. 76: 697-706

21. Chan FK, Siegel RM, Zacharias D, Swofford R, Holmes KL, Tsien RY and Lenardo MJ (2001) Fluorescence resonance energy transfer analysis of cell 
surface receptor interactions and signaling using spectral variants of the green fluorescent protein. PG Cytometry 44: 361-368

22. Siegel RM, Chan FK, Zacharias DA, Swofford R, Holmes KL, Tsien RY and Lenardo MJ (2000) Measurement of molecular interactions in living cells by fluorescence resonance energy transfer between variants of the green fluorescent protein. PG - PL1. Sci. STKE 2000: PL1

23. Periasamy A and Day RN (1999) Visualizing protein interactions in living cells using digitized GFP imaging and FRET microscopy. Methods Cell Biol. 58: 293-314

24. Juo P, Woo MS, Kuo CJ, Signorelli P, Biemann HP, Hannun YA and Blenis J (1999) FADD is required for multiple signaling events downstream of the receptor Fas. Cell Growth Differ. 10: 797-804

25. Chen CY, Juo P, Liou JS, Li CQ, Yu Q, Blenis J and Faller DV (2001) The recruitment of Fas-associated death domain/caspase-8 in Ras-induced apoptosis. Cell Growth Differ. 12: 297-306

26. Scaffidi C, Fulda S, Srinivasan A, Friesen C, Li F, Tomaselli KJ, Debatin KM, Krammer PH and Peter ME (1998) Two CD95 (APO-1/Fas) signaling pathways. EMBO J. 17: 1675-1687
27. Muppidi JR and Siegel RM (2004) Ligand-independent redistribution of Fas (CD95) into lipid rafts mediates clonotypic T cell death. Nat. Immunol. 5: 182-189

28. Thomas LR, Stillman DJ and Thorburn A (2002) Regulation of Fas-associated death domain interactions by the death effector domain identified by a modified reverse two-hybrid screen. J. Biol. Chem. 277: 34343-34348

29. Yang JK, Wang L, Zheng L, Wan F, Ahmed M, Lenardo MJ and Wu H (2005) Crystal Structure of MC159 Reveals Molecular Mechanism of DISC Assembly and FLIP Inhibition. Mol. Cell 20: 939-949

30. Duke TA and Bray D (1999) Heightened sensitivity of a lattice of membrane receptors. Proc. Natl. Acad. Sci. USA 96: 10104-10108

31. Acehan D, Jiang X, Morgan DG, Heuser JE, Wang X and Akey CW (2002) Three-dimensional structure of the apoptosome: implications for assembly, procaspase-9 binding, and activation. Mol. Cell 9: 423-432

32. Martinon F, Burns $\mathrm{K}$ and Tschopp J (2002) The inflammasome: a molecular platform triggering activation of inflammatory caspases and processing of prolL-beta. Mol. Cell 10: 417-426

33. Tinel A and Tschopp J (2004) The PIDDosome, a protein complex implicated in activation of caspase-2 in response to genotoxic stress. Science 304: 843-846 